\title{
Application of NMR-based metabolomics to the study of marine bivalves
}

Aru, Violetta; Engelsen, Søren Balling; Cesare Marincola, Flaminia

Published in:

Proceedings of the XIII International Conference on the Applications of Magnetic Resonance in Food Science

DOI:

$10.1255 / \mathrm{mrfs} .8$

Publication date:

2016

Document version

Publisher's PDF, also known as Version of record

Document license:

CC BY

Citation for published version (APA):

Aru, V., Engelsen, S. B., \& Cesare Marincola, F. (2016). Application of NMR-based metabolomics to the study of marine bivalves. In Proceedings of the XIII International Conference on the Applications of Magnetic Resonance in Food Science: a collection of peer-reviewed papers from the conference (pp. 39-43) https://doi.org/10.1255/mrfs.8 


\title{
Application of NMR-based metabolomics to the study of marine bivalves
}

\author{
Violetta Aru ${ }^{\star a}, b$, Søren Balling Engelsen and Flaminia Cesare Marincola ${ }^{b}$ \\ ${ }^{a}$ Chemometrics \& Analytical Technology, Department of Food Science, University of Copenhagen, Rolighedsvej 26, 1958, Frederiksberg C, \\ Denmark. Corresponding Author: violetta@food.ku.dk; se@food.ku.dk \\ ${ }^{\mathrm{b}}$ Department of Chemical and Geological Sciences, Cittadella Universitaria di Monserrato, University of Cagliari, S.S. 554 Bivio Sestu, \\ 09042, Monserrato, Italy. Email: flaminia@unica.it
}

This paper reviews the results of three studies on the application of high-field proton Nuclear Magnetic Resonance $\left({ }^{1} \mathrm{H}\right.$ NMR) spectroscopy and metabolomics to the assessment of different issues related to marine bivalves. The outcomes of such applications yielded insights into multiple aspects concerning the impact of cold storage on shellfish quality and the effect of natural and anthropogenic stressors on bivalve metabolic profiles. In the first work, the effect of different cold storage conditions on the Mediterranean mussel Mytilus galloprovincialis (Lamarck, 1819) was investigated, revealing substantial time-related changes both in the metabolic profiles and microbial loads of stored mussels. In the second study, the seasonal change and reproductive cycle were demonstrated to be the leading causes behind the fluctuation in the amino acid and carbohydrate contents in the European clam Ruditapes decussatus (Linnaeus, 1758). Finally, NMR metabolomics was applied for assessing the effect of lead and zinc short-term exposure on the metabolic profiles of two clam species, Ruditapes decussatus and Ruditapes philippinarum (Adams \& Reeve, 1850). The results evidenced a sensible metabolic response upon metal pollution, pointing out a main variability in the content of amino acids and organic osmolytes in relation to both metal nature and bivalve species.

\section{Introduction}

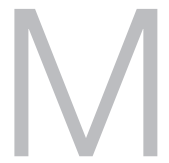

etabolomics is a scientific discipline focused on the study of the overall dynamic set of the endogenous and exogenous low molecular weight metabolites $(<1.5 \mathrm{KDa})$ which includes various organic species such as amino acids, fatty acids, nucleic acids, carbohydrates, organic acids, vitamins, polyphenols and small lipids (metabolome $)^{1}$. Being the downstream product of gene expression, as well as the ultimate response to disease or environmental influences, the metabolome can be regarded as a reliable snapshot of the physiological state of an organism. Thanks to the development of powerful analytical methods, nowadays metabolomics plays an important role in gaining biological insights towards the influence of internal (genetic and developmental) and external (environmental) factors on phenotypes. Different analytical platforms are used to study the metabolome of biological systems but Nuclear Magnetic Resonance (NMR) spectroscopy plays a prominent role in metabolomics due to its capability to provide a robust chemical overview ${ }^{2}$. If coupled with chemometrics, it becomes a suitable tool for the analysis of complex biological samples, making NMR-based metabolomics a powerful approach for assessing the biochemical responses arising from typical/atypical physiological conditions.

Metabolomics has been widely demonstrated to be a highthroughput technique in several scientific disciplines from microbiology to clinical medicine ${ }^{3}$. In the field of food sciences, the "metabolomics approach" has been used for addressing several issues related to nutrition, safety, and food quality ${ }^{4-7}$. In particular, due to the remarkable nutritional properties of seafood products, aspects concerning fish as foodstuffs are increasingly being investigated by metabolomics ${ }^{8}$. Among different applications, NMR-based metabolomics was demonstrated to be a viable tool for assessing the effect of different storage conditions on fish and fish-derived products $^{9-10}$ as well as for distinguishing fish from different aquaculture systems ${ }^{11}$. In contrast, metabolomics studies of bivalve molluscs, which are important commercial resources in the aquaculture industry, are still very scarce. In this paper, an overview on the application of NMR-based metabolomics to the study of marine bivalves is given, with a focus on food and environmental sciences.

\section{Materials and methods Samples collection}

Bivalve specimens were collected in a local seafood market Cagliari (Sardinia, Italy). After collection, the samples were transported to the laboratory in portable coolers, at approximately $4^{\circ} \mathrm{C}$. In the laboratory, bivalve samples were immediately shucked and deprived of the digestive gland and the intestine. The soft tissue of the specimens was then put into insulated sterile plastic boxes and stored at $-80^{\circ} \mathrm{C}$ before metabolites extraction ${ }^{12}$.

\section{NMR spectra acquisition}

${ }^{1} \mathrm{H}-\mathrm{NMR}$ spectra were acquired with a Varian Unity Inova 500 spectrometer (Agilent Technologies, CA, USA) operating at $499.84 \mathrm{MHz}$ at $300 \mathrm{~K}$. For each spectrum, 256 scans were collected into $32 \mathrm{~K}$ data points over a spectral width of $6000 \mathrm{~Hz}$, with a $45^{\circ}$ pulse, an acquisition time of $1.5 \mathrm{~s}$, and a relaxation delay of $4 \mathrm{~s}$. The solvent (water) residual signal was suppressed by applying a presaturation technique with a low power radiofrequency irradiation for $1.5 \mathrm{~s}$. An exponential function corresponding to $0.3 \mathrm{~Hz}$ was applied to each Free Induction Decay (FID) before Fourier transformation as well as a zero-filling to $65 \mathrm{k}$ data points. 


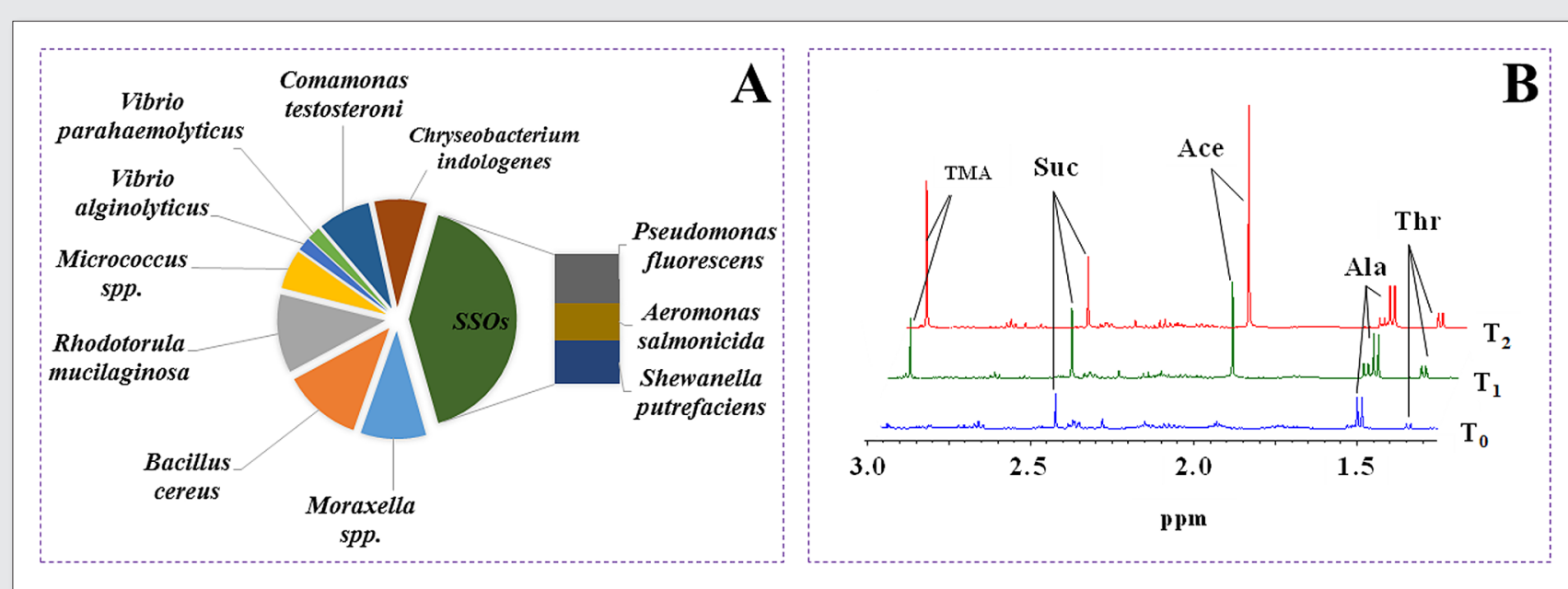

Figure 1. Average distribution of the microbial species isolated from $M$. galloprovincialis samples stored at $4^{\circ} \mathrm{C}(\mathrm{A})$; Expansion of the aliphatic region of three representative ${ }^{1} \mathrm{H}$ NMR spectra of the hydrosoluble extract of stored mussels (B). $T_{0}$ : fresh sample; $T_{1}: 2$ days of storage; $T_{2}: 6$ days of storage.

\section{Pre-processing and chemometric analysis of the NMR data}

Prior to chemometric analysis, NMR spectra were manually phased and baseline corrected using MestReNova (Version 8.1, Mestrelab Research SL). Spectral chemical shift referencing was also performed in all spectra on the TSP $\mathrm{CH}_{3}$ signal at $0.00 \mathrm{ppm}$. SIMCA version 13.0 (Umetrics, Umea, Sweden) and Matlab (Mathworks, MA) were used for the Multivariate Analysis (MVA) of the spectral dataset. In the latter case, the NMR spectra were corrected for small chemical shift misalignments using the icoshift algorithm ${ }^{13}$. Spectra were then normalized ${ }^{14}$ and Pareto scaled ${ }^{15}$. The chemometric methods applied for the analysis of the spectral dataset included unsupervised and supervised approaches. Principal Component Analysis $(\mathrm{PCA})^{16}$ was used to data overview, while Partial Least Square Discriminant Analysis (PLS-DA) ${ }^{17}$ and Orthogonal Partial Least Square Discriminant Analysis (O-PLS-DA) ${ }^{18}$ were applied aiming at detecting class-related biomarkers.

Metabolite-Metabolite Correlation Analysis (MMCA) was performed in order to investigate the interdependencies between metabolites and systemic (pattern) changes. Correlation analysis is widely applied to the study of systems biology and has been demonstrated to be a valuable tool also in the field of metabolomics where it is commonly applied to explore the rdynamics amongst metabolites ${ }^{19}$.

\section{Results and discussion}

\section{NMR-based metabolomics for spoilage detection}

One of the main issues of seafood marketing is the ease with which fish and shellfish undergo deterioration after death. Bivalve mollusks, in particular, are amongst the most perishable seafood: the lack of less digestive connective tissues and the elevated water activity lead to a faster degradation by microorganisms ${ }^{20}$. Although the role of bacteria in seafood spoilage is well recognized, data on the possible link between the metabolic profiles and the microbial activity are still lacking. In this work, metabolomics was combined with microbiological analysis in order to explore possible correlations between the biochemical alterations of the $M$. galloprovincialis samples, stored at $0{ }^{\circ} \mathrm{C}$ and $4^{\circ} \mathrm{C}$ for a period of 10 and 6 days, respectively, and the microbial loads over the storage time.
As far as the microbiological analysis is concerned, psychrotrophic Gram-negative bacteria were found to be the dominant pool of microorganisms in stored mussels. They were mainly represented by Pseudomonas fluorescens, Aeromonas salmonicida and Shewanella putrefaciens species. These bacteria, referred to as "Specific Spoilage Organisms" or SSOs ${ }^{21}$, are able to produce Volatile Organic Compounds (VOCs), such as TriMethylAmine (TMA), responsible for off-flavours and taste associated with spoiled seafood. Among other species, the yeast Rhodotorula mucilaginosa and Bacillus cereus, characterized by a noteworthy proteolytic activity, were particularly abundant in the isolates at both temperatures. Overall, both mesophilic and psychrotrophic viable bacterial counts significantly increased throughout the storage period in samples stored at $4^{\circ} \mathrm{C}$ while significant lower counts were observed at refrigerated storage at $0^{\circ} \mathrm{C}$. By way of example, in Figure $1 \mathrm{~A}$ the average distribution of the microbial species isolated from samples of $M$. galloprovincialis stored at $4^{\circ} \mathrm{C}$ is reported.

The chemometric analysis allowed the identification of several putative biomarkers of spoilage in the NMR spectra of stored mussels. Overall, the most remarkable metabolic changes at both storage temperatures, although with different rates, were linked to proteolysis, glycogen-lysis, glucose metabolism and the bacterial production of volatile amines which were manifested in an increase in the relative concentrations of free amino acids (i.e. alanine and threonine), organic acids (i.e. acetate, lactate, succinate), and TMA, respectively. The abovementioned spoilage process is exemplified in Figure 1B in which the representative spectra of the hydrosoluble extract of fresh mussels and those stored at $4^{\circ} \mathrm{C}$ are reported.

These results demonstrate that NMR-based metabolomics is a valuable tool for the assessment of the quality of stored shellfish providing a basis for further investigations aimed at identifying innovative biomarkers of seafood freshness.

\section{Effect of the seasonal change and reproductive cycle on the metabolic profile of Ruditapes decussatus}

In this study ${ }^{1} \mathrm{H}-\mathrm{NMR}$ metabolomics was applied as analytical method for the assessment of the effect of the seasonal change and reproductive cycle on the metabolome of the European clam Ruditapes decussatus, collected in a commercial site in the Santa 
Gilla lagoon during different months of the year, from May 2013 to July 2014. This clam is one of the typical constituents of the macrobenthos biocenosis of the site and represents one of the main products of the Santa Gilla fishing activity. Temperature and salinity were seasonally measured at the clams harvesting site in order to investigate possible links between the seasonal variations of these chemical physical parameters and the clams metabolic profile.

PCA applied on the NMR spectral dataset of the hydrosoluble extract of clams allowed the identification of several metabolites potentially related with the harvesting season. In particular, the content of free amino acid was found to vary according to the sampling period. The concentrations of these molecules is known to be directly affected by processes of cell water regulation following osmotic stress and is frequently used as an indicator for a species capability to cope with salinity changes ${ }^{22}$. Therefore, salinity and temperature acclimation might be the leading causes behind the observed changes in the relative amounts of free amino acids. In agreement with literature ${ }^{23}$, the seasonal fluctuations in salinity and temperature, measured in the harvesting site, co-varied with alanine and glycine in the clams' metabolome. Furthermore, a second remarkable variation in clams' biochemical composition was observed in the relative distribution of carbohydrates. Carbohydrates are considered to be the main energy source in marine bivalves ${ }^{24}$ since they cover a prominent role in gametes formation as well as in adults maintaining during nutritive stress periods, such as the reproductive cycle. In $R$. decussatus the reproductive cycle comprises two phases: a resting phase (autumn-winter) and the gametogenesis (spring-summer) which includes ripeness and spawning ${ }^{25}$. In this study the highest concentrations of carbohydrates were found during the resting phase (autumn), when energetic resources are

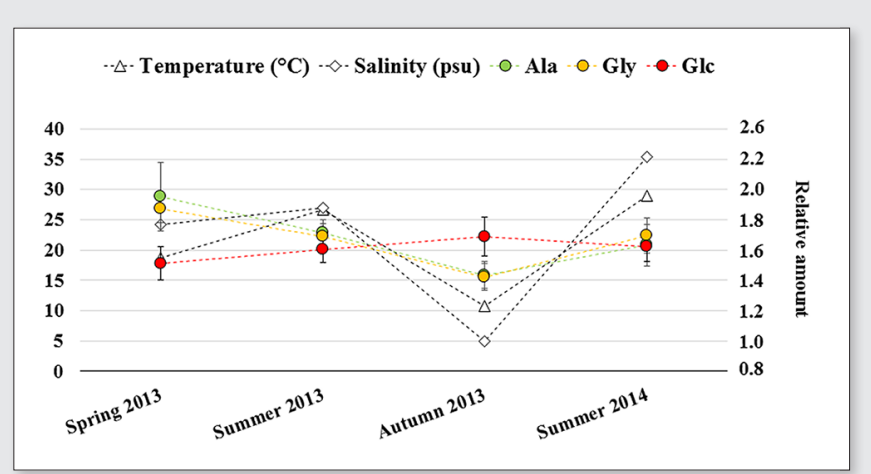

Figure 2. Seasonal mean values of water temperature $(\Delta)$ and salinity $(\diamond)$ and fluctuations in the relative amount of alanine (green circle), glycine (yellow circle) and glucose (red circle) in wild samples of $R$. decussatus harvested in Santa Gilla lagoon. Chemical-physical parameters were measured in the clams harvesting area with a multiparametric probe (Hanna H 9828).

accumulated in preparation for spring gametogenesis, while minimum concentrations occurred at maturity (spring-summer), when the metabolic demand is higher. In Figure 2 the seasonal mean value of water temperature and salinity and the fluctuations in the relative amount of alanine, glycine and glucose are reported.

The application of NMR-based metabolomics in the present work has provided valuable information on the seasonal cyclic variations in the carbohydrate and amino acid content in the European clam, and represents a step forward towards a more comprehensive molecular characterization of this marine bivalve, which potentially could be of great interest in the field of human nutrition.

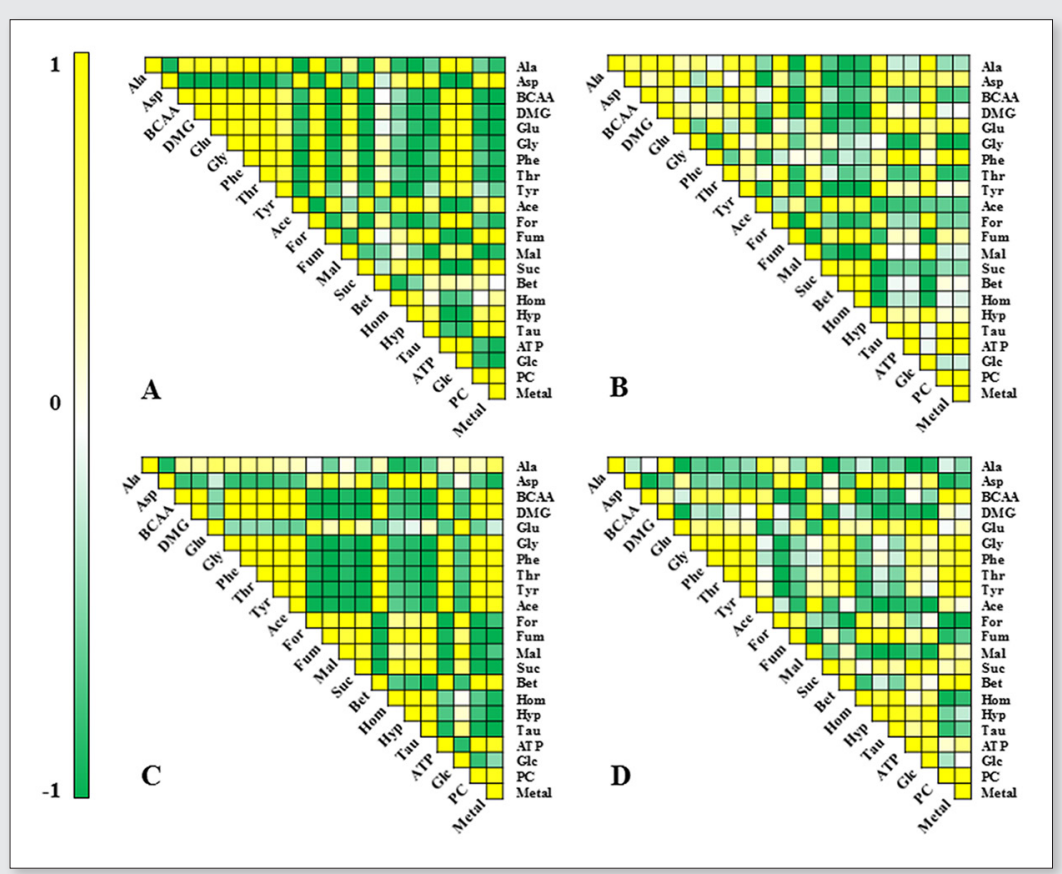

Figure 3. Metabolite-metabolite correlation matrices (heat maps) for lead and zinc treated $R$. decussatus ( $\mathrm{A}$ and $\mathrm{B}$, respectively) and lead and zinc treated R. philippinarum ( $\mathrm{C}$ and D, respectively). The heat maps are colored according to Pearson's correlation coefficient (r). Deeper colors indicate higher positive (yellow) or negative (green) correlation coefficients. Keys: Ace=acetate; Ala=alanine; Asp=aspartate; ATP=adenosine triphosphate; BCAA=branched chain amino acids; Bet=betaine; DMG=dimethylglycine; For=formate; Fum=fumarate; Glc=glucose; Glu=glutamate; Gly=glycine; Hom=homarine; Hyp=hypotaurine; Mal=malonate; PC=phosphocholine; Phe=phenylalanine; Suc=succinate; Tau=taurine; Thr=threonine; Tyr=tyrosine. 


\section{Metabolic responses of the clams Ruditapes decussatus and Ruditapes philippinarum to heavy metal exposure}

As persistent inorganic pollutants, heavy metals are one of the major harmful toxicants for the marine environment ${ }^{26}$. The accumulation of such contaminants in aquatic species leads to concentrations that are several orders of magnitude higher than those of the average water $^{27}$, and thus become of concern to public health when bioaccumulated in edible animals. Besides the negative effects linked with the ingestion of toxic trace elements, metal accumulation leads to the re-balance of the so called "compatible solutes" (i.e. organic osmolytes, small carbohydrates, polyols, amino acids and derivatives) $^{22}$, thus affecting the biochemical composition of aquatic organisms. In this work, NMR-based metabolomics was applied to assess the biochemical responses of two clam species to $48 \mathrm{~h}$ exposure to increasing concentrations of lead nitrate (10,40, 60, and $100 \mu \mathrm{g} / \mathrm{L})$ and zinc chloride (20,50, 100, and $150 \mu \mathrm{g} / \mathrm{L})$, under laboratory conditions. A comprehensive overview on the initial pollution state of the samples and the occurrence of bioaccumulation was achieved by complementing the NMR data with a direct heavy metal detection in clams by Inductively Coupled Plasma Optical Emission Spectrometry (ICP-OES).

PCA was performed on the ${ }^{1} \mathrm{H}-\mathrm{NMR}$ spectra of the aqueous extracts of clams to seek valid metabolic signatures, potentially translatable into metal poisoning. Following metal treatment, clams' metabolic responses appeared to be both species- and metalspecific. Metal exposure in $R$. philippinarum induced an increase in the relative amount of free amino acids (threonine, tyrosine and phenylalanine), while it decreased the content of organic osmolytes. The opposite behaviour was exhibited by $R$. decussatus. Organic osmolytes and amino acids are typically accumulated in aquatic invertebrates for counteracting osmotic perturbations and maintaining cell volume, suggesting metal exposure as the leading cause behind the observed metabolic fingerprint. Further information on the effect of heavy metal pollution on clams' metabolic fingerprint was achieved by investigating metabolite-metabolite correlations, calculated in terms of Pearson's correlations. Lead treated samples showed a higher number of strong positive and negative correlations suggesting a more robust effect of lead poisoning on clams' metabolic profiles.

Despite the sensitivity of the metabolomics approach in revealing short-time metal-induced stress, it is interesting to note that no significant metal accumulation was monitored in the same specimens by using ICP-OES analysis.

These results confirm NMR-based metabolomics as a promising tool for the identification of putative biomarkers of contaminantinduced stress and evidence the utility of metabolomics in finding a biomarker contour to be used for seafood quality control.

\section{Conclusions}

The presented findings have provided valuable information concerning different issues related to three species of marine bivalves: the mussel Mytilus galloprovincialis and the clams Ruditapes decussatus and Ruditapes philippinarum. In particular, it has been demonstrated that the use of ${ }^{1} \mathrm{H}$ NMR spectroscopy in combination with multivariate data analysis is a feasible approach for assessing the effect of different cold storage conditions on the mussels' hydrosoluble components, as well as for the identification of molecular biomarkers of environmental and anthropogenic stress in marine clams. These results represent a step forward towards a more complete biochemical characterization of $M$. galloprovincialis, $R$. decussatus and $R$. philippinarum and will open the way for further investigations aimed at achieving deeper insights on bivalves' bioecological framework.

\section{Acknowledgments}

Violetta Aru gratefully acknowledges Sardinia Regional Government for the financial support of her PhD scholarship (P.O.R. Sardegna F.S.E. Operational Programme of the Autonomous Region of Sardinia, European Social Fund 2007-2013 - Axis IV Human Resources, Objective I.3, Line of Activity 1.3.1).

\section{References}

1. O. Fiehn, "Metabolomics - the link between genotypes and phenotypes", Plant Mol Biol. 48, 155-171 (2002). doi: http://dx.doi. org/10.1023/A:1013713905833

2. W.B. Dunn and D.I. Ellis, "Metabolomics: Current analytical platforms and methodologies", TrAC. 24, 285-294 (2005). doi: http://dx.doi. org/10.1016/j.trac.2004.11.021

3. S.P. Putri, Y. Nakayama, F. Matsuda, T. Uchikata, S. Kobayashi, A. Matsubara and E. Fukusaki, "Current metabolomics: Practical applications". J Biosci Bioeng. 115, 579-589 (2013). doi: http://dx.doi.org/10.1016/j. jbiosc. 2012.12.007

4. J.M. Cevallos-Cevallos, J.I Reyes-De-Corcuera, E. Etxeberria, M.D. Danyluk and G.E. Rodrick, "Metabolomic analysis in food science: a review", Trends Food Sci Tech. 20, 557-566 (2009). doi: http://dx.doi. org/10.1016/j.tifs.2009.07.002

5. D.S. Wishart, "Metabolomics: applications to food science and nutrition research", Trends Food Sci Tech. 19, 482-493 (2008). doi: http://dx.doi. org/10.1016/j.tifs.2008.03.003

6. B. Khakimov, G. G rdeniz and S.B. Engelsen, "Trends in the application of chemometrics to foodomics studies", Acta Aliment. 44, 4-31 (2015). doi: http://dx.doi.org/10.1556/AAlim.44.2015.1.1

7. F. Savorani, M.A. Rasmussen, M.S. Mikkelsen and S.B. Engelsen, "A primer to nutritional metabolomics by NMR spectroscopy and chemometrics", Food Res Int. 54, 1131-1145 (2013). doi: http://dx.doi. org/10.1016/j.foodres.2012.12.025

8. L.M Samuelsson and D.G.J Larsson, "Contributions from metabolomics to fish research", Mol Biosyst. 4, 974-979 (2008). doi: http://dx.doi. org/10.1039/b804196b

9. G. Picone, S.B. Engelsen, F. Savorani, S. Testi, A. Badiani and F. Capozzi, "Metabolomics as a powerful tool for molecular quality assessment of the fish Sparus aurata", Nutrients. 3, 212-227 (2011). doi: http:// dx.doi.org/10.3390/nu3020212

10. E. Locci, C. Piras, S. Mereu, F. Cesare Marincola and P. Scano, "1H NMR metabolite fingerprint and pattern recognition of mullet (Mugil cephalus) bottarga", J Agr Food Chem. 59, 9497-9505 (2011). doi: http://dx.doi. org/10.1021/jf2012979

11. F. Savorani, G. Picone, A. Badiani, P. Fagioli, F. Capozzi and S.B. Engelsen, "Metabolic profiling and aquaculture differentiation of gilthead sea bream by 1H NMR metabonomics", Food Chem. 120, 907-914 (2010). doi: http://dx.doi.org/10.1016/j.foodchem.2009.10.071

12. J. Folch, M. Lees, G.H.S Stanley, "A simple method for the isolation and purification of total lipids from animal tissues", J Biol Chem. 226, 497509 (1957). PMID: 13428781

13. F. Savorani, G. Tomasi and S.B. Engelsen, "icoshift: A versatile tool for the rapid alignment of 1D NMR spectra". J Magn Reson. 202, 190-202 (2010). doi: http://dx.doi.org/10.1016/j.jmr.2009.11.012 
14. A. Craig, O. Cloarec, E. Holmes, J.K. Nicholson and J.C. Lindon, "Scaling and normalization effects in NMR spectroscopic metabonomic data sets". Anal Chem. 78, 2262-2267 (2006). doi: http://dx.doi.org/10.1021/ ac0519312

15. B. Worley and R. Powers, "Multivariate Analysis in Metabolomics". Curr Metabolomics. 1, 92-107 (2012). doi: http://dx.doi.org/10.2174/22132 35X11301010092

16. S. Wold, K. Esbensen and P. Geladi, "Principal component analysis", Chemometr Intell Lab. 2, 37-52 (1987). doi: http://dx.doi. org/10.1016/0169-7439(87)80084-9

17. M. Barker and W. Rayens, "Partial least squares for discrimination", J Chemometr. 17, 166-173 (2003). doi: http://dx.doi.org/10.1002/ cem.785

18. M. Bylesjo, M. Rantalainen, O. Cloarec, J.K Nicholson, E. Holmes and J. Trygg, "OPLS discriminant analysis : combining the strengths of PLSDA and SIMCA classification", J chemometr. 20, 341-351 (2007). doi: http://dx.doi.org/ 10.1002/cem.1006

19. R. Steuer, J. Kurths, O. Fiehn and W. Weckwerth, " Observing and interpreting correlations in metabolomic networks”, Bioinformatics. 19, 10191026 (2003). doi: http://dx.doi.org/10.1093/bioinformatics/btg120

20. E. Goulas, I. Chouliara, E. Nessi, M.G. Kontominas and I.N. Savvaidis, "Microbiological, biochemical and sensory assessment of mussels (Mytilus galloprovincialis) stored under modified atmosphere packaging", J Appl Microbiol. 98, 752-760 (2005). doi: http://dx.doi.org/10.1111/ j.1365-2672.2004.02512.x
21. P. Dalgaard, "Modelling of microbial activity and prediction of shelf life for packed fresh fish", Intl J Food Microbiol. 26, 305-317 (1995). doi: http:// dx.doi.org/10.1016/0168-1605(94)00136-T

22. P.H. Yancey, "Organic osmolytes as compatible, metabolic and counteracting cytoprotectants in high osmolarity and other stresses". J Exp Biol. 208, 2819-2830 (2005). doi: http://dx.doi.org/10.1242/jeb.01730

23. S. Kube, A. Sokolowski, J.M. Jansen and D. Schiedek, "Seasonal variability of free amino acids in two marine bivalves, Macoma balthica and Mytilus spp ., in relation to environmental and physiological factors", Comp Biochem Phys A. 147, 1015-1027 (2007). doi: http://dx.doi. org/10.1016/i.cbpa.2007.03.012

24. D.I. Zandee, J.H. Kluytmans, W. Zurburg and H. Pieters, "Seasonal variations in biochemical composition of Mytilus edulis with reference to energy metabolism and gametogenesis", Neth J Sea Res. 14, 1-29 (1980). doi: http://dx.doi.org/10.1016/0077-7579(80)90011-3

25. J. Ojea, A.J. Pazos, D. Martínez, S. Novoa, J.L. Sánchez and M. Abad, "Seasonal variation in weight and biochemical composition of the tissues of Ruditapes decussatus in relation to the gametogenic cycle". Aquaculture. 238, 451-468 (2004). doi: http://dx.doi.org/10.1016/j.aquaculture.2004.05.022

26. J. S. Gray, "Biomagnification in marine systems: the perspective of an ecologist ". Mar Pollut Bull. 45, 46-52 (2002). doi: http://dx.doi. org/10.1016/S0025-326X(01)00323-X

27. S. Casas, J.L. Gonzales, B. Andral, and D. Cossa, "Relation between metal concentration in water and metal content of marine mussel (Mytilus galloprovincialis): impact of physiology", Environ Toxicol Chem. 27, 1543-1552 (2008). doi: http://dx.doi.org/10.1897/07-418.1 